\title{
Real-time quantitative RT-PCR detection of circulating tumor cells from breast cancer patients
}

\author{
MAOWEN GUO ${ }^{1,2}$, XIAOTIAN LI ${ }^{1}$, SHAOHUA ZHANG ${ }^{4}$, HUA SONG $^{4}$, WENHUI ZHANG ${ }^{2}$, XUEYI SHANG ${ }^{2,4}$, \\ YULING ZHENG ${ }^{2}$, HUA JIANG ${ }^{2}$, QINGYU LV ${ }^{2}$, YONGQIANG JIANG ${ }^{2}$ and HUAIJIE HAO ${ }^{2,3}$ \\ ${ }^{1}$ School of Pharmacy, Zhengzhou University, Zhengzhou, Henan 450001; ${ }^{2}$ State Key Laboratory of Pathogen \\ and Biosecurity, Institute of Microbiology and Epidemiology, Academy of Military Medical Sciences, Beijing 100071; \\ ${ }^{3}$ CAS Key Laboratory of Pathogenic Microbiology and Immunology, Institute of Microbiology, Chinese Academy of Sciences, \\ Beijing 100101; ${ }^{4}$ Affiliated Hospital of Academy of Military Medical Sciences, Beijing 100071, P.R. China
}

Received August 22, 2014; Accepted September 22, 2014

DOI: $10.3892 /$ ijo.2014.2732

\begin{abstract}
Circulating tumor cells (CTCs) were recognized as novel tumor biomarker for prognostic and predictive purposes in various cancers. Various detection technologies and devices have been developed to enumerate and characterize CTCs. Most of those approaches are based on the positive enrichment strategy and immunocytological techniques. However, the sensitivity of these approaches proved to be limited in metastatic tumors and the detection of early tumor cell dissemination was problematic. In the present study, we developed a novel CTC detection method by real-time RT-PCR technique in combination of negative enrichment strategy. The developed enrichment approach could recover more than $75 \%$ of spiked breast cancer cells from peripheral blood. The detection limit of duplex real-time RT-PCR assay using KRT19 and ERBB2 as targeted genes was consistently one breast tumor cell. Moreover, CTC detection by duplex real-time RT-PCR assay had higher detection sensitivity than that by immunostaining, especially in early breast cancer. In summary, the results of the present study indicated the potential clinical utilities of CTCs identification on breast cancer by duplex real-time RT-PCR in combination with negative enrichment.
\end{abstract}

Correspondence to: Dr Huaijie Hao, CAS Key Laboratory of Pathogenic Microbiology and Immunology, Institute of Microbiology, Chinese Academy of Sciences, No. 1 Beichen West Road, Chaoyang, Beijing 100101, P.R. China

E-mail: haohj@im.ac.cn

Professor Yongqiang Jiang, State Key Laboratory of Pathogen and Biosecurity, Institute of Microbiology and Epidemiology, Academy of Military Medical Sciences, No. 20 Dongda Street, Fengtai, Beijing 100071, P.R. China

E-mail: jiangyq@bmi.ac.cn

Key words: circulating tumor cells, real-time RT-PCR, breast cancer, negative enrichment

\section{Introduction}

Breast cancer is the most common cancer in women worldwide and the leading cause of death from cancer among women globally. According to the GLOBOCAN 2012 data (1), World Health Organization International Agency for Research on Cancer, there were nearly 1.7 million new cases diagnosed in 2012 which is $\sim 12 \%$ of all new cancer cases and $25 \%$ of all cancers in women. Fortunately, the increasing application of effective therapies such as adjuvant medical therapy made it possible for the mortality rate to decline over the last decades. However, it also poses a challenge for clinicians to avoid over-treatment, insufficient treatment or incorrect treatment due to lack of useful prognostic, diagnostic and monitoring biomarkers (2).

Routine clinical therapies for breast cancer are based on the classical clinical and pathological features from immunohistochemistry evaluations, radiologic evaluations and serum tumor markers. Unfortunately, these features are incapable of providing enough information on the ongoing metastasis as early as possible for predicting the clinical outcome with high accuracy and reproducibility. As an emerging tumor biomarker, circulating tumor cell (CTC) analysis is a promising new diagnostic field for micrometastasis, although it depends on the emergence of increasingly advanced and sensitive technologies to isolate and characterize human CTCs (3). Multiple independent studies have demonstrated that CTCs can be recognized as novel tumor biomarkers for prognostic and predictive purposes in metastatic breast cancer (4-8).

Various detection technologies and devices have been developed to enumerate and characterize CTCs (9-13), but no gold standard could define the absolute accuracy, sensitivity and specificity in detecting CTCs. For the isolation of CTCs, a variety of currently used methods were based on various properties of CTCs compared with leukocytes and broadly divided into two isolation/enrichment strategies (positive or negative strategy). The positive strategy was based on epithelial cell adhesion molecule (EpCAM) or cytokeratin expression profile or cell size, while the negative strategy was developed by targeting and removing the normal blood cells. For the detection of CTCs, immunocytological approaches 
Table I. Oligonucleotide primer and probe sequences used in the present study.

\begin{tabular}{|c|c|c|c|c|c|c|}
\hline $\begin{array}{l}\text { Gene } \\
\text { symbol }\end{array}$ & $\begin{array}{c}\text { Accession } \\
\text { number }\end{array}$ & Primer-probe & Sequence $\left(5^{\prime}-3^{\prime}\right)$ & $\begin{array}{l}\mathrm{Tm} \\
\left({ }^{\circ} \mathrm{C}\right)\end{array}$ & $\begin{array}{c}\text { Amplicon } \\
\text { size (bp) }\end{array}$ & Fluorophore-quencher \\
\hline \multirow[t]{3}{*}{ ERBB2 } & NM_004448 & Primer_F & CCTGGCCGTGCTAGACAATGG & 58.5 & 138 & \\
\hline & & Primer_R & GGGTTCCGCTGGATCAAGACC & 58.2 & & \\
\hline & & Probe & CGCTGAACAATACCA & 69 & & VIC-MGB-NFQ \\
\hline \multirow[t]{3}{*}{ KRT19 } & NM_002276 & Primer_F & CAGATCGACAATGCCCGTCTGG & 59 & 149 & \\
\hline & & Primer_R & TGCATCTCCAGGTCGGTCCTG & 59 & & \\
\hline & & Probe & AGATGACTTCCGAAC & 69 & & FAM-MGB-NFQ \\
\hline \multirow[t]{3}{*}{ EPCAM } & Nm_002354 & Primer_F & GCTGGCCGTAAACTGCTTTGTG & 58.5 & 115 & \\
\hline & & Primer_R & TGCCTTCATCACCAAACATTTGGC & 58.6 & & \\
\hline & & Probe & AATCGTCAATGCCAG & 69 & & VIC-MGB-NFQ \\
\hline \multirow[t]{3}{*}{ MUC1 } & NM_002456 & Primer_F & GGTGCTGGTCTGTGTTCTGG & 58.9 & 136 & \\
\hline & & Primer_R & GTACTCGCTCATAGGATGGTAGG & 58.3 & & \\
\hline & & Probe & CCATTGTCTATCTCATTGC & 69 & & NED-MGB-NFQ \\
\hline
\end{tabular}

and molecular techniques such as RT-PCR are the most widely used. To date, the CellSearch ${ }^{\circledR}$ system is the only approved assay by FDA for prognosis in metastatic breast, prostate and colon cancer which captures the CTCs by immunomagnetic beads coated with antibodies targeting EpCAM and identify CTCs by fluorescent probes for intracellular protein cytokeratin 8, 18 and 19. However, the CellSearch ${ }^{\circledR}$ system was shown to have a lower sensitivity in early breast cancer (14).

In the present study, we developed a new CTC detection approach based on negative enrichment strategy and realtime quantitative RT-PCR technique. The approach applied in this study for breast cancer CTC detection showed both high sensitivity and specificity, especially in early breast cancer.

\section{Materials and methods}

Ethical statement. Human blood samples were obtained from the Affiliated Hospital of Academy of Military Medical Sciences (Beijing, China). All patients and healthy donors enrolled in the present study signed the Consent Forms approved by the Ethics Review Committee of the Academy of Military Medical Sciences.

Materials. Fetal bovine serum (FBS), RPMI-1640 media, DMEM media, TRIzol reagent, glycogen, Ambion TUBRO DNA-free kit, SuperScript ${ }^{\mathrm{TM}}$ III First-Strand Synthesis system for RT-PCR, TaqMan ${ }^{\circledR}$ Universal Master Mix II kit, rabbit anti-cytokeratin (Pan) polyclonal antibody, Alexa Fluor 594 labeled goat anti-rabbit IgG antibody, Dynabeads CD45, Hoechst 33342 and Mitotracker ${ }^{\circledR}$ Red CMXRos were obtained from Life Technologies (Grand Island, NY, USA). T-25 cell culture flasks, 50- and 15-ml tubes were purchased from Corning Inc. (Corning, NY, USA). Ficoll-Paque Plus was purchased from GE Healthcare (Uppsala, Sweden). BD Vacutainer ${ }^{\circledR}$ evacuated blood collection tube with acid citrate dextrose anticoagulant were from Becton-Dickinson (Franklin Lakes, NJ, USA).

Cell lines and culture conditions. Human breast cancer cell line SK-BR-3, MCF-7, MDA-MB-453, ZR 75-1, human promyelocytic leukemia cell line HL-60, human Brukitt's lymphoma cell line Raji and human T-cell leukemia cell line Jurkat were obtained from the Cell Resource Center (IBMS, CAMS/PUMC, Beijing, China). The SK-BR-3, ZR 75-1, HL-60, Raji and Jurkat cell lines were maintained in RPMI1640 medium supplemented with $10 \%$ FBS, the MCF-7 and MDA-MB-453 cell lines were maintained in DMEM medium supplemented with $10 \%$ FBS. All cell cultures were maintained at $37^{\circ} \mathrm{C}$ in a humidified atmosphere with $5 \% \mathrm{CO}_{2}$.

Tumor cell enrichment. We developed the tumor cell enrichment method based on negative enrichment and density gradient centrifuge strategy that updated our previously published method (15). Briefly, $1 \mathrm{ml}$ of peripheral blood collected in BD Vacutainer tube was diluted with PBS to $15 \mathrm{ml}$ and subsequently incubated with $100 \mu \mathrm{l}$ Dynabeads CD45 for $30 \mathrm{~min}$ at room temperature with gentle tilting and rotation. Then, the whole blood sample was carefully layered on $7.5 \mathrm{ml}$ Ficoll-Paque Plus in a $50-\mathrm{ml}$ centrifuge tube, followed by spinning at $350 \mathrm{x}$ for $5 \mathrm{~min}$ at $4^{\circ} \mathrm{C}$. Supernatants were transferred into a centrifuge tube followed by spinning at $650 \mathrm{x} \mathrm{g}$ for $5 \mathrm{~min}$. Cell pellet was stained in glass slide and subsequently subjected to fluorescent microscope observation, or extracted mRNA and performed real-time RT-PCR detection.

Primers and probes design. Primers and probes (Table I) were designed with AlleleID 6.0 (Premier Biosoft) and synthesized by Life Technologies Corp. (Beijing, China). Nucleotide sequences used for design of probe-primers were retrieved from NCBI database and the designed probe-primers were aligned by BLAST to confirm gene specificity. 
RNA isolation and complementary DNA synthesis. Total RNA from enriched cells was extracted using TRIzol reagent according to the manufacturer's manual. To enhance the precipitation of RNA from small quantity of cell samples, glycogen (final concentration $250 \mu \mathrm{g} / \mathrm{ml}$ ) was added to the cell lysate before phase separation. The contaminating DNA from RNA preparation was removed using Ambion ${ }^{\circledR}$ TURBO DNA-free kit according to the manufacturer's protocol.

Complementary DNA (cDNA) synthesis was performed using the SuperScript ${ }^{\mathrm{TM}}$ III First-Strand Synthesis system for RT-PCR. Briefly, the following conditions were performed in a total volume of $20 \mu \mathrm{l}: 1 \mu \mathrm{l}$ oligo(dT) primer and $1 \mu \mathrm{l}$ $10 \mathrm{mM}$ dNTP mix were mixed with $8 \mu \mathrm{l}$ mRNA, incubated for $5 \mathrm{~min}$ at $65^{\circ} \mathrm{C}$ for $5 \mathrm{~min}$, and then placed on ice for at least $1 \mathrm{~min}$. Then, $10 \mu \mathrm{l}$ of cDNA synthesis mix was added [2 $\mu 1$ 10X RT buffer, $4 \mu 125 \mathrm{mM} \mathrm{MgCl}_{2}, 2 \mu 10.1 \mathrm{M}$ DTT, $1 \mu 1 \mathrm{RNaseOUT}^{\mathrm{TM}}(40 \mathrm{U} / \mu \mathrm{l})$, SuperScript ${ }^{\mathrm{TM}}$ III RT (200 U/ $\mu 1)$ ] to each RNA/primer mixture and incubated for $50 \mathrm{~min}$ at $50^{\circ} \mathrm{C}$ and the reactions at $85^{\circ} \mathrm{C}$ for 5 min was terminated. The sample was chilled on ice and $1 \mu \mathrm{l}$ of RNase $\mathrm{H}$ was added to the tube and incubated for $20 \mathrm{~min}$ at $37^{\circ} \mathrm{C}$. The product of cDNA synthesis reaction was stored at $-20^{\circ} \mathrm{C}$ or used for realtime PCR immediately.

Multiplex real-time PCR. The real-time PCR was performed using the TaqMan ${ }^{\circledR}$ Universal Master Mix II kit with the StepOnePlus ${ }^{\mathrm{TM}}$ real-time PCR instrument. The PCR experiments were performed according to the protocol and cycling conditions outlined in the manual. The final concentrations of each primer and probe in the real-time PCR reaction were 0.4 and $0.2 \mu \mathrm{M}$, respectively if not otherwise indicated.

Immunostaining and identification of enriched CTCs. Immunostaining of CTCs were performed as previously described with some modifications (15). Briefly, enriched cells were fixed by $2 \%$ paraformaldehyde on glass slides and then permeabilized with $0.1 \%$ Triton $\mathrm{X}-100$, followed by incubation with rabbit anti-cytokeratin (Pan) polyclonal antibody for $1 \mathrm{~h}$. Slides were washed three times with PBS, followed by incubated with Alexa Fluor 594 labeled goat anti-rabbit IgG antibody for $1 \mathrm{~h}$. After being washed three times with PBS, slides were applied with mounting media containing DAPI (Vector Laboratories) and subsequently subjected to image analysis using laser confocal scanning microscope FV1000 (Olympus). For identification of CTC, each positive CTC had to meet the following criteria: cell size $>4 \mu \mathrm{m}$, cells were intact with round to oval morphology with visible DAPI stained nucleus, and positive for cytokeratin staining.

Spiking study. Several validation experiments by spiking study were performed to establish the accuracy of the method to detect cancer cells in blood. To validate the recovery rate of the enrichment method, liver human breast cancer cells were labeled with Hoechst 33342 and Mitotracker ${ }^{\circledR}$ Red CMXRos for $1 \mathrm{~h}$. Different numbers of cells were counted with a fluorescent microscope (BX53; Olympus, Tokyo, Japan) and spiked into $1 \mathrm{ml}$ blood from healthy donor. The samples were treated by the enrichment isolation procedure and recovered cancer cells were enumerated using a fluorescent microscope (BX53;

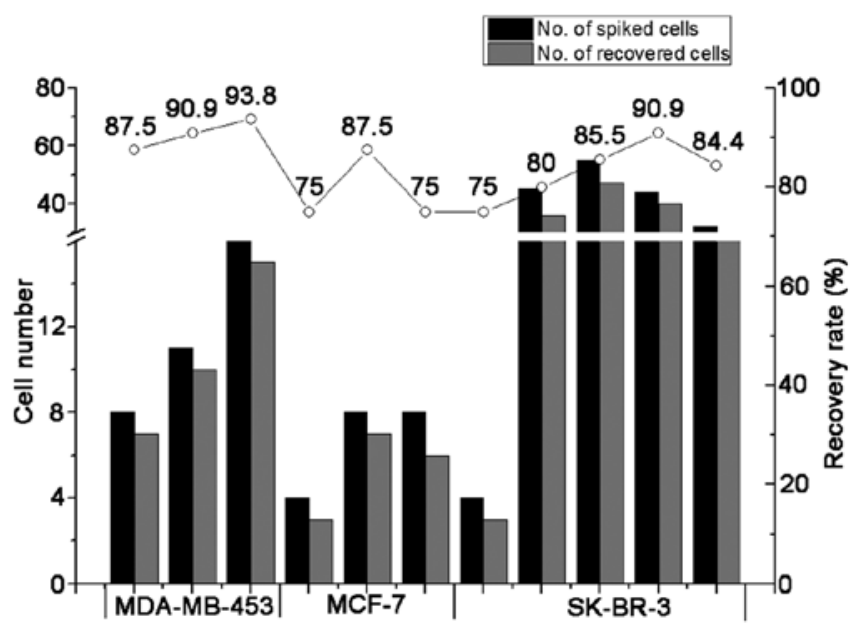

Figure 1. Validation of recovery efficiency of the new enrichment strategy by spiking study. Live human breast cancer cells MDA-MB-453, MCF-7, SK-BR-3 were labeled with Mitotraker ${ }^{\circledR}$ Red CMXRos and Hoechst 33342 , respectively. The labeled cells were counted under a microscope and spiked into $1 \mathrm{ml}$ bloods from healthy donor, respectively. After enrichment, the recovered labeled cells were enumerated under a microscope.

Olympus) by an observer in a blinded manner. To validate the accuracy of real-time RT-PCR, liver breast cancer cells were spiked into different blood cells or cell lines (Raji, HL-60, Jurkat and isolated leukocytes) respectively, and the mixed samples were detected the expression of tumor marker genes by real-time RT-PCR assay.

Patients and specimens. Fifteen healthy donors and sixteen breast cancer patients were enrolled in the present study. Peripheral blood $(2 \mathrm{ml})$ was drawn from the median cubital vein into a BD Vacutainer tube (with acid citrate dextrose anticoagulant), and to avoid potential epithelial cell contamination, the first $2 \mathrm{ml}$ of blood were discarded before each collection of blood samples. The blood sample was equally divided into two aliquots and processed immediately. Then, the enriched CTCs from two aliquots were identified by immunostaining or real-time RT-PCR analysis, respectively.

\section{Results}

Validation of recovery efficiency of the new enrichment strategy by spiking study. A series of blinded spiking studies were performed to validate the developed enrichment method of detecting breast cancer cells in blood. Live human breast cancer cell lines MDA-MB-453, MCF-7 and SK-BR-3 were labeled with Mitotracker ${ }^{\circledR}$ Red CMXRos and Hoechst 33342 as described in Materials and methods. After counting under a microscope, the labeled cells were spiked into $1 \mathrm{ml}$ fresh peripheral blood collected from healthy donors to simulate the blood of tumor patients. The blood samples were treated with the enrichment procedure and the recovered tumor cells were enumerated using a fluorescent microscope (BX53; Olympus) by an observer in a blinded manner. As shown in Fig. 1, $>75 \%$ cells (range from 75 to $93.8 \%$, mean $84.1 \%$ ) could be recovered from spiked MDA-MB-453, MCF-7 and SK-BR-3 cells. These results indicated that our enrichment method was able to efficiently enrich and recover spiked 
Table II. Ct values of ERBB2, KRT19 and EpCAM transcripts in serial dilutions of RNA extracted from the cell line SK-BR-3, MCF-7 and ZR 75-1.

\begin{tabular}{|c|c|c|c|c|c|c|c|}
\hline \multirow[b]{2}{*}{ Gene } & \multirow[b]{2}{*}{ Cell no. } & \multicolumn{2}{|c|}{ SK-BR-3 } & \multicolumn{2}{|c|}{ MCF-7 } & \multicolumn{2}{|c|}{ ZR 75-1 } \\
\hline & & $\mathrm{Ct}($ mean $\pm \mathrm{SD})$ & $\begin{array}{l}\% \text { positive } \\
\text { replicates }\end{array}$ & $\mathrm{Ct}($ mean $\pm \mathrm{SD})$ & $\begin{array}{l}\% \text { positive } \\
\text { replicates }\end{array}$ & $\mathrm{Ct}($ mean $\pm \mathrm{SD})$ & $\begin{array}{l}\% \text { positive } \\
\text { replicates }\end{array}$ \\
\hline \multirow[t]{6}{*}{ ERBB2 } & 10,000 & $18.9 \pm 1.28$ & 100 & $25.58 \pm 0.14$ & 100 & $20.96 \pm 1.02$ & 100 \\
\hline & 1,000 & $22.99 \pm 1.83$ & 100 & $29.28 \pm 0.14$ & 100 & $24.54 \pm 1.28$ & 100 \\
\hline & 100 & $26.91 \pm 1.96$ & 100 & $32.30 \pm 0.08$ & 100 & $28.01 \pm 1.54$ & 100 \\
\hline & 10 & $29.96 \pm 1.92$ & 100 & $33.77 \pm 0.09$ & 100 & $30.97 \pm 1.74$ & 100 \\
\hline & 1 & $32.98 \pm 1.57$ & 100 & $34.29 \pm 0.01$ & 100 & $32.96 \pm 1.94$ & 100 \\
\hline & 0.1 & $34.59 \pm 1.40$ & 100 & $34.24 \pm 0.10$ & 100 & $33.37 \pm 2.04$ & 100 \\
\hline \multirow[t]{6}{*}{ KRT19 } & 10,000 & $17.98 \pm 0.88$ & 100 & $22.28 \pm 1.80$ & 100 & $17.59 \pm 3.62$ & 100 \\
\hline & 1,000 & $22.64 \pm 1.08$ & 100 & $27.33 \pm 2.33$ & 100 & $22.18 \pm 4.91$ & 100 \\
\hline & 100 & $26.75 \pm 1.34$ & 100 & $30.99 \pm 2.01$ & 100 & $26.12 \pm 5.07$ & 100 \\
\hline & 10 & $30.08 \pm 1.23$ & 100 & $33.65 \pm 2.00$ & 100 & $29.72 \pm 4.82$ & 100 \\
\hline & 1 & $33.64 \pm 1.09$ & 100 & $34.27 \pm 2.25$ & 100 & $32.52 \pm 3.18$ & 100 \\
\hline & 0.1 & $37.74 \pm 2.34$ & 86 & $35.96 \pm 0.00$ & 50 & $34.26 \pm 2.29$ & 67 \\
\hline \multirow[t]{6}{*}{ EpCAM } & 10,000 & $20.94 \pm 1.78$ & 100 & $19.84 \pm 0.40$ & 100 & $16.63 \pm 1.10$ & 100 \\
\hline & 1,000 & $25.18 \pm 1.49$ & 100 & $23.53 \pm 0.29$ & 100 & $20.35 \pm 1.52$ & 100 \\
\hline & 100 & $28.30 \pm 0.38$ & 93 & $27.11 \pm 0.04$ & 100 & $23.90 \pm 1.84$ & 100 \\
\hline & 10 & $30.71 \pm 0.85$ & 86 & $30.87 \pm 0.29$ & 100 & $27.51 \pm 2.09$ & 100 \\
\hline & 1 & $32.05 \pm 1.38$ & 86 & $34.05 \pm 0.37$ & 100 & $31.53 \pm 1.94$ & 100 \\
\hline & 0.1 & $33.29 \pm 2.06$ & 71 & $34.30 \pm 0.17$ & 100 & $32.88 \pm 2.67$ & 83 \\
\hline
\end{tabular}

breast cancer cells from peripheral blood, with a high degree of accuracy.

Development of multiplex real-time RT-PCR detection of breast CTCs. For the real-time RT-PCR detection of breast CTCs, MUC1, EpCAM, ERBB2 and KRT19 were selected as candidate marker genes. To evaluate if the selected genes were appropriate marker genes for RT-PCR assay, we first detected the expression of these genes in different breast tumor cells and human blood cells. The results showed that all these selected genes were expressed highly in breast tumor cell lines SK-BR-3, MCF-7 and ZR 75-1 (data not shown). However, the expression of MUC1 gene could also be detected in Raji, Jurkat and isolated leukocytes when the cell number was $>50,000$ (Fig. 2). These results indicated that MUC1 may not be a suitable marker gene for detection of CTCs in peripheral blood.

Then, we tried to determine the sensitivity of the real-time RT-PCR detection of breast CTCs. For this, serial dilutions of total RNA from breast cancer cell line SK-BR-3, MCF-7 and ZR 75-1 were performed to detect ERBB2, KRT19 and EpCAM gene, respectively. The detection limit using ERBB2 or KRT19 (Table II) as targeted gene was consistently one tumor cell RNA of SK-BR-3, MCF-7 or ZR 75-1, which indicated that ERBB2 and KRT19 could potentially detect a single breast cancer cell. But, the sensitivity of real-time RT-PCR detection of CTCs using EpCAM gene as targeted gene was not consistent in all tested cell lines (Table II). Based on these results, we chose the ERBB2 and KRT19 as the marker genes to develop the real-time RT-PCR detection of breast CTCs.
Table III. Comparation of amplification efficiency of KRT19 and ERBB2 in a duplex assay and corresponding singleplex assays.

\begin{tabular}{llll}
\hline Gene & Assay & Slope & $\mathrm{R}^{2}$ \\
\hline KRT19 & Duplex & -3.54 & 0.973 \\
& Singleplex & -3.88 & 0.977 \\
ERBB2 & Duplex & -3.51 & 0.98 \\
& Singleplex & -3.61 & 0.925 \\
\hline
\end{tabular}

To test if the contaminated leukocytes after negative enrichment affect the sensitivity and specificity of real-time RT-PCR detection of CTCs, various numbers of SK-BR-3 cells were spiked into a serial diluted leukocytes and detected by real-time RT-PCR assay. As shown in Fig. 3, the Ct number of breast CTCs without contaminated leukocytes was exactly equal to that of equal breast CTCs with the different numbers of contaminated leukocytes, which indicated the contaminating leukocytes did not affect the amplification of ERBB2 and KRT19 genes in breast CTCs.

Based on the above results, we decided to develop the duplex real-time RT-PCR detection of breast CTCs using KRT19 and ERBB2 primer-probe sets. Next, we compared the sensitivity of the duplex and singleplex assays by detecting total RNA of 10-fold serial dilutions of SK-BR-3 (from $10^{5}$ to $\left.10^{-1}\right)$. For the duplex reactions, the primers and probes were 
A

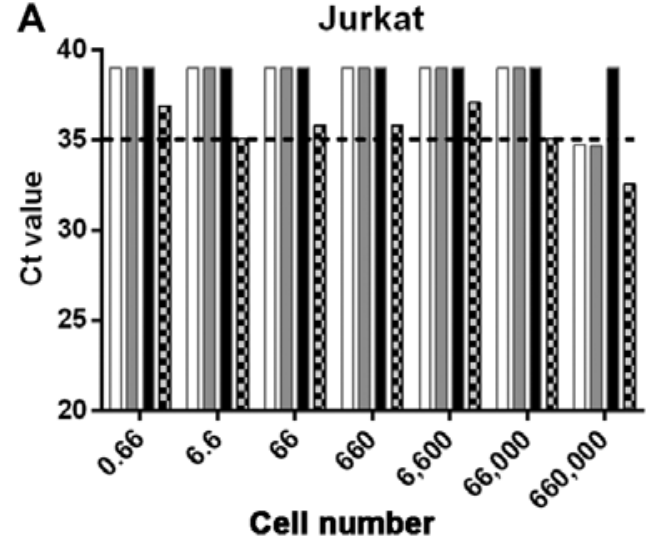

B

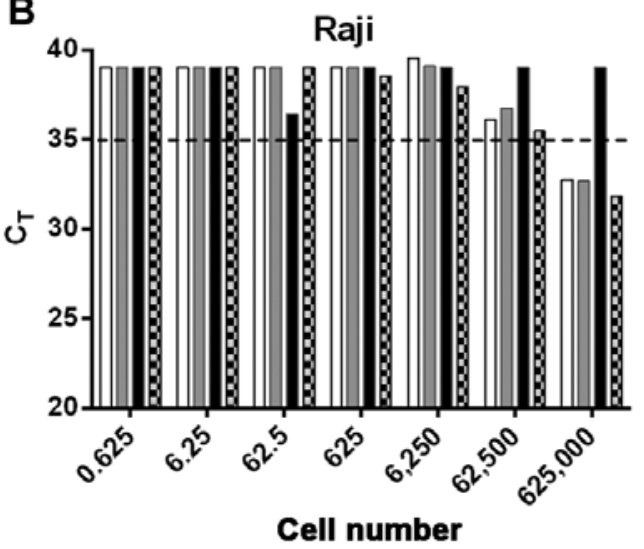

C

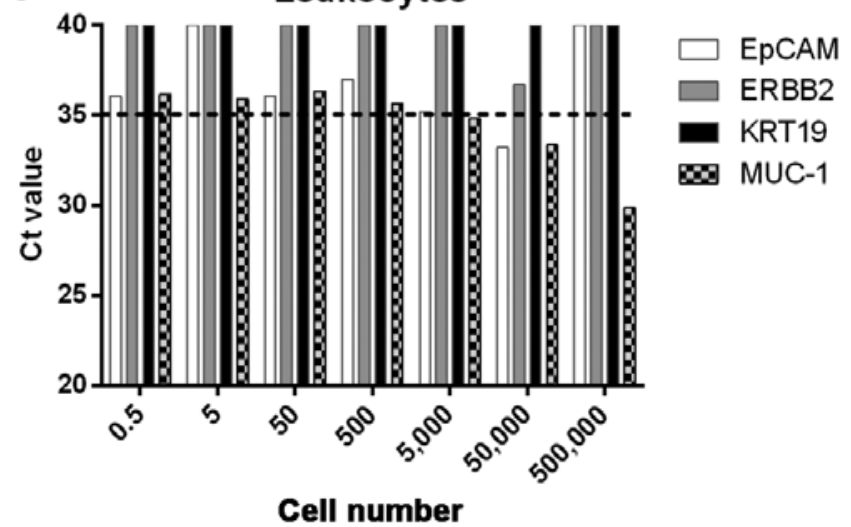

Figure 2. Gene expression of EpCAM, ERBB2, KRT19 and MUC1 in cell line (A) Jurkat, (B) Raji and (C) leukocytes. To evaluate the potential contribution of gene transcript levels derived from human blood cells, transcript levels of EpCAM, ERBB2, KRT19 and MUC1 were detected with real-time RT-PCR assay.

each used at $0.2 \mu \mathrm{M}$, and for the singleplex reactions, the probe concentration was set at $0.2 \mu \mathrm{M}$, while the primer concentration was set at $0.4 \mu \mathrm{M}$. The amplification plots achieved with duplex assay overlapped with those from the singleplex assays (data not shown), and equivalent $\mathrm{Ct}$ value, slope and $\mathrm{R}^{2}$ were achieved for each gene regardless of whether a duplex or a singleplex assay was performed (Table III). These results indicated that the developed duplex assay had equivalent sensitivity with singleplex assays.
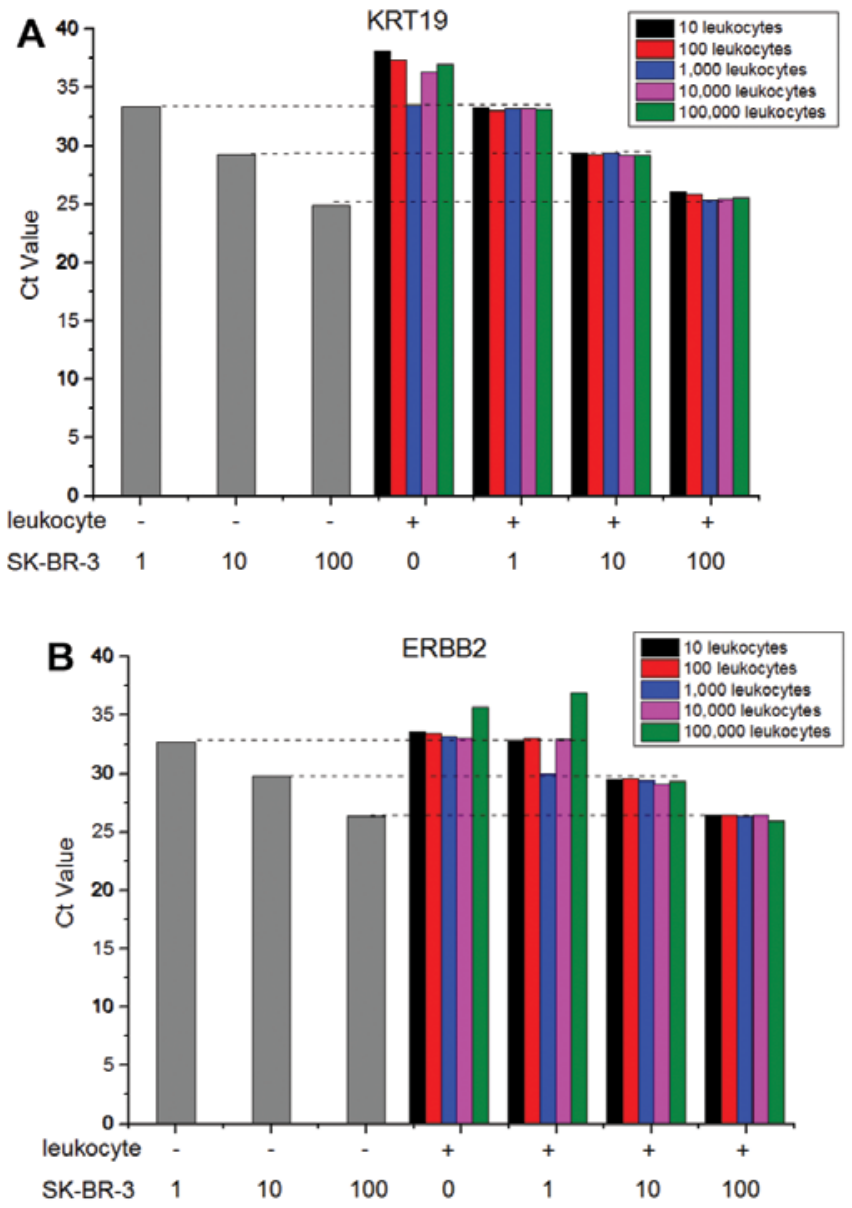

Figure 3. Influence of contaminated leukocytes on the amplification of (A) KRT19 and (B) ERBB2 in breast CTCs. Different number of SK-BR-3 cells $(1,10$ and 100$)$ were spiked into different number of leukocytes $(10$, to $100,000)$, respectively. After extraction of total RNA, the KRT19 or ERBB2 transcript in different samples was amplified, respectively.

Enrichment and detection of CTCs from breast cancer patients. Having proved that the breast tumor cells could be recovered efficiently by negative enrichment and detected quantitatively by duplex real-time RT-PCR assay, we applied the approaches to the detection of CTCs in the whole blood samples. In the present study, 15 healthy donors (Table IV) and 16 breast cancer patients (Table V) including 2 stage I, 6 stage II, 3 stage III and 5 stage IV were enrolled. Tumornode-metastasis (TNM) staging of breast cancer patients was performed according to seventh edition of cancer staging manual by the American Joint Committee on Cancer (AJCC). Blood samples from breast cancer patients were collected before drug therapy and subsequently subjected to enrichment and CTC counting. To evaluate the results of real-time RT-PCR assay, enriched CTCs from another aliquot of those blood samples were also identified by immunostaining with anti-cytokeratin (pan) antibody and counted under fluorescence microscopy (Table V). Fig. 4 shows some representative enriched breast circulating tumor cells which were intact with round to oval morphology with visible DAPI stained nucleus and positive for anti-cytokeratin (pan) staining.

For the quantification of enriched CTCs by real-time RT-PCR assay, total RNAs extracted from a serial of concen- 

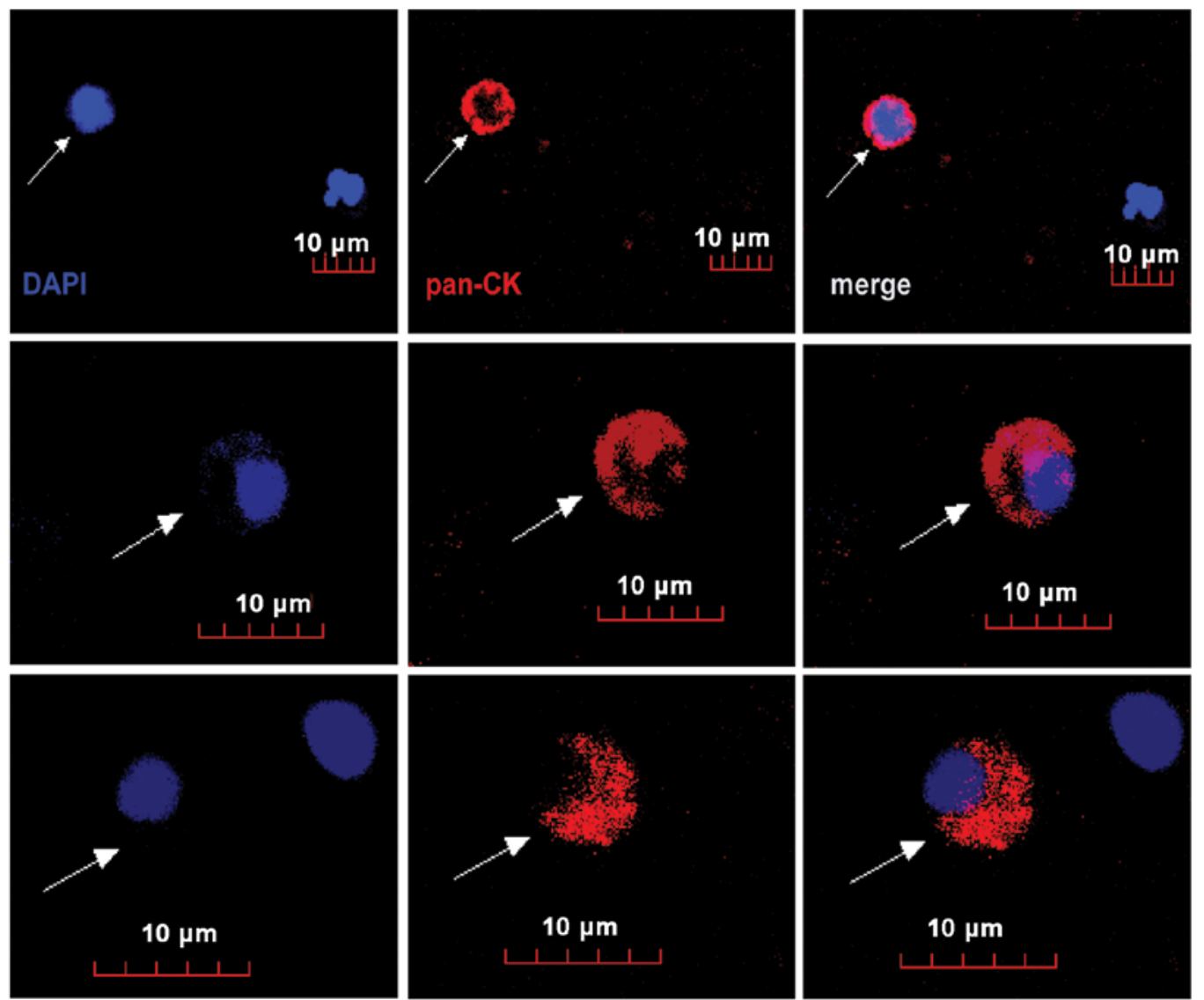

Figure 4. Images of breast CTCs from peripheral blood of patient BCA27. Enriched CTCs were subjected to immunofluorescent staining with anti-cytokeratin (pan) antibody (red) and nuclear counterstain DAPI (blue). The stained CTCs were visualized with confocal microscope FV1000 (Olympus).

Table IV. Quantification of CTCs of blood samples from healthy people.

\section{Healthy CTCs identified CTCs idenfied CTCs identified} sample no. by ICC by KRT19 by ERBB2

\begin{tabular}{llll}
\hline HD01 & 0 & 0 & 0 \\
HD02 & 0 & 0 & 0 \\
HD03 & 0 & 0 & 1 \\
HD04 & 0 & 0 & 0 \\
HD05 & 0 & 0 & 0 \\
HD06 & 0 & 0 & 0 \\
HD07 & 0 & 2 & 0 \\
HD08 & 0 & 1 & 0 \\
HD09 & 0 & 1 & 0 \\
HD10 & 0 & 0 & 1 \\
HD11 & 0 & 0 & 0 \\
HD12 & 0 & 0 & 1 \\
HD13 & 0 & 2 & 1 \\
HD14 & 0 & 0 & 0 \\
HD15 & 0 & 0 & 0 \\
\hline
\end{tabular}

trations of SK-BR-3 cells served as a template for an external calibration curve to calculate the quantity of breast CTCs. Among the 15 healthy donors, 8 people were detected with
1 or 2 CTCs by KRT19 and/or ERBB2 genes. For this reason, we set 5 as a cut-off value of positive CTCs for all real-time RT-PCR assay. For the immunocytochemistry staining, no healthy donors could be detected with CTC. Thus, a positive patient was defined as one whose CTC count in $1 \mathrm{ml}$ blood was $>2$ for immunocytochemistry staining.

Distributions of positive CTC cells among healthy donors and patients are demonstrated in Fig. 5 and summarized in Table VI. According to the cut-off value, the positive CTC detection rate was zero in healthy donors by real-time RT-PCR assay or immunocytochemistry staining. For immunocytochemistry staining, the positive detection rates for all histologic cancers according to stage I-II, III and IV were 28.5, 75 and $80 \%$, respectively. For real-time RT-PCR assay by KRT19 gene, the positive detection rates for stage I-II, III and IV were 71, 100 and $80 \%$, respectively. For real-time RT-PCR assay by ERBB2 gene, the positive detection rates for stage I-II, III and IV were 57, 100 and $100 \%$. Combining the results of realtime RT-PCR assay by KRT19 and ERBB2 gene together, the positive detection rate for stage I-II, III and IV reached 85.7, 100 and $100 \%$, respectively. These results indicated that breast CTCs detection by duplex real-time RT-PCR assay had higher detection sensitivity than that by immunostaining.

\section{Discussion}

In the present study, we developed a novel circulating breast tumor cell detection approach using duplex real-time RT-PCR 


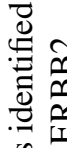

$\bigcup_{0}^{\infty}$

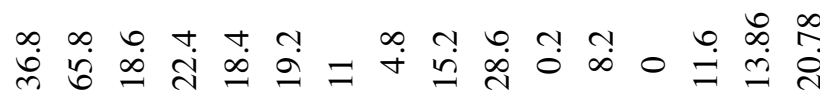

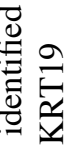

已

先

:

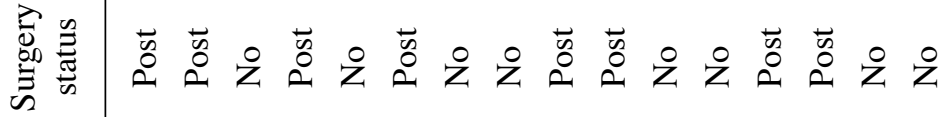

赵薳 尺

$\stackrel{\infty}{\diamond}$

$\succsim$

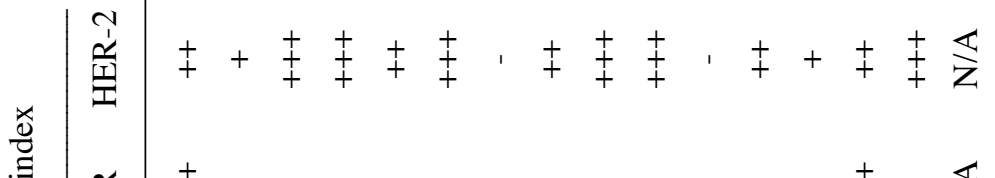

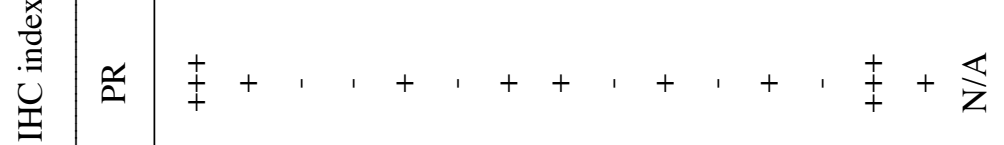

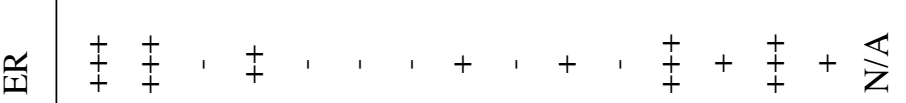

$\underset{\circlearrowright}{\mathscr{D}}$

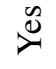

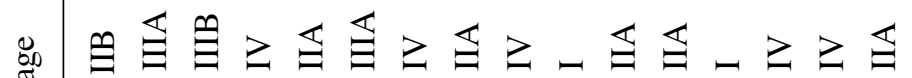

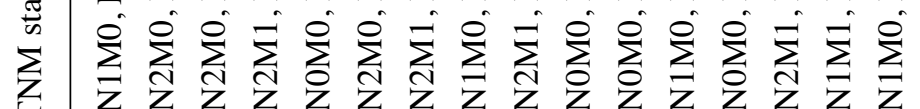

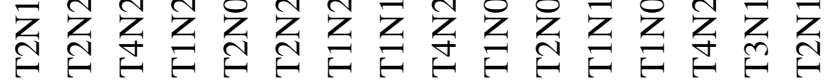

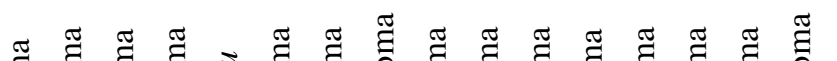

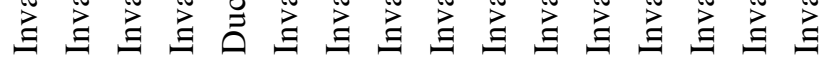



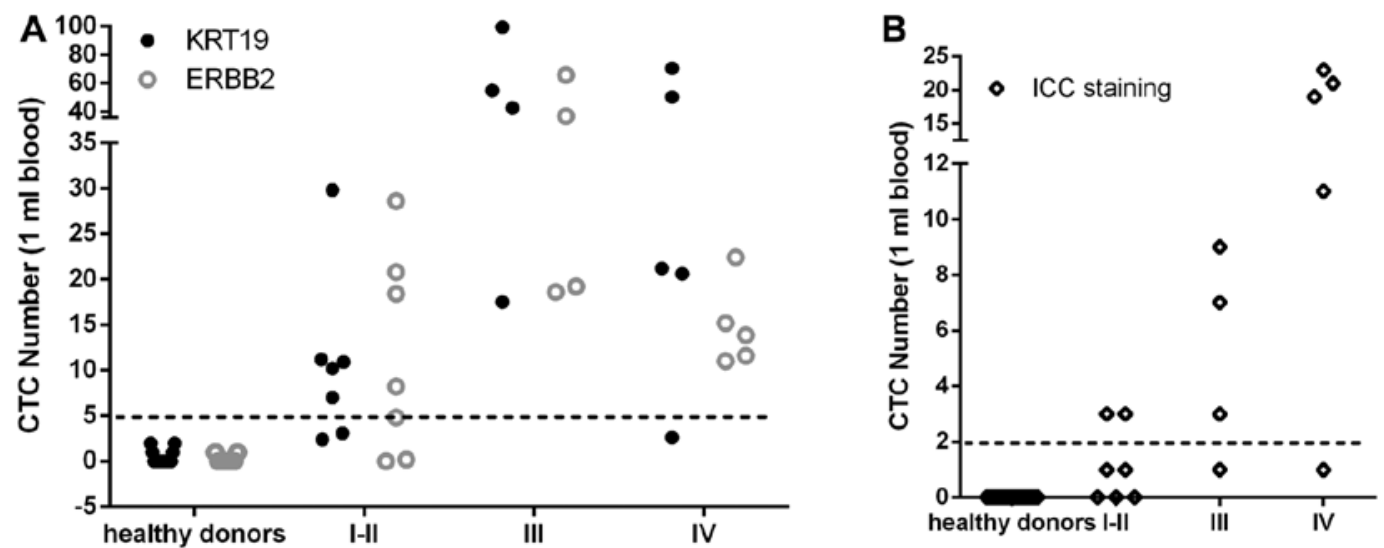

Figure 5. Distribution of CTCs identified by real-time RT-PCR assay and immunocytochemistry staining. Fifteen healthy donors and 16 breast cancer patients before subjected to drug therapy were enrolled and CTCs were identified in peripheral blood by (A) duplex real-time RT-PCR assay and (B) immunocytochemistry staining, respectively. For real-time RT-PCR assay, a cut-off value of $>5$ cancer cells per $1 \mathrm{ml}$ blood was defined as positive. For ICC staining, the positive cut-off value was set $>2$ cancer cells per $1 \mathrm{ml}$ blood.

Table VI. Analysis of CTC detection by real-time PCR and immunostaining.

\begin{tabular}{lcccc}
\hline & & \multicolumn{2}{c}{ CTCs $>5$} & CTCs $>2$ \\
\cline { 3 - 4 } $\begin{array}{l}\text { Pathological } \\
\text { type }\end{array}$ & Number & $\begin{array}{c}\text { Identified by KRT19 } \\
\text { Positive (\%) }\end{array}$ & $\begin{array}{c}\text { Identified by ERBB2 } \\
\text { Positive (\%) }\end{array}$ & $\begin{array}{c}\text { Identified by ICC staining } \\
\text { Positive (\%) }\end{array}$ \\
\hline Healthy donors & 15 & $0(0)$ & $0(0)$ & $0(0)$ \\
I-II & 7 & $5(71)$ & $4(57)$ & $2(28.5)$ \\
III & 4 & $4(100)$ & $4(100)$ & $3(75)$ \\
IV & 5 & $4(80)$ & $5(100)$ & $4(80)$ \\
\hline
\end{tabular}

technique in combination with negative enrichment strategy. Our results showed that the developed negative enrichment approach could efficiently enrich and recover breast tumor cells from peripheral blood with a high degree of accuracy, and the duplex real-time RT-PCR assay using KRT19 and ERBB2 as targeted genes could consistently detect one breast tumor cell even in the environment containing relative large quantities of contaminating leukocytes.

Since CTCs are rare cells in peripheral blood, isolation of CTCs represents a major technological challenge and enrichment prior to the actual detection procedure could improve the sensitivity of CTCs detection. The most widely used CTC isolation technologies rely on positive enrichment by antibody-based capture of CTCs which express epithelial cell surface markers such as the epithelial cell adhesion molecule (EpCAM) that are absent from normal leukocytes. But, not all CTCs express the EpCAM antigen. Moreover, EpCAM expression tends to change dynamically during the epithelialmesenchymal transition (EMT) process in the metastatic cascade (16-18). To avoid the bias of selecting cells by virtue of EpCAM expression, negative enrichment by removing leukocytes has been advocated which has the potential to purify CTCs irrespective of presumed cell surface markers and the advantages such as keeping the CTCs in an intact/untargeted form over positive enrichment in isolating rare cells (18-21).

To determine the performance of the system in the negative enrichment process, the overall level of enrichment, recovery rate and sensitivity were the common quantitative measures. Different negative enrichment approaches based on immunomagnetic separation demonstrated different overall level of enrichment, ranging from 2.7 to $5.66 \mathrm{log}$ for fresh peripheral blood, and different recovery rate by spiking experiment, range from 36 to $85 \%$ (22). Although a completely negative selection methodology might achieve a higher enrichment performance, it also might reduce the recovery rate (22). In the present study, we tried to perform the negative enrichment process with as few steps as possible to balance between enrichment level and recovery rate. We used a density gradient centrifugation step instead of the red blood cell lysis step to reduce the possible harm and loss to CTCs $(23,24)$. Here, we achieved an average $3 \log$ enrichment (data not shown), and such concentration is sufficient to perform further RT-PCR analysis of CTCs with less background contamination. Furthermore, we achieved a high recovery rate (range from 75 to $93.8 \%$, mean $84.1 \%$ ) to increase the overall sensitivity of the CTCs detection.

Another major challenge for CTC identification was the prevailing difficulty of finding an mRNA marker which could distinguish normal expression in blood from that due to the presence of CTCs. Several mRNA markers have been used for RT-PCR-based detection of CTCs and evaluated for their sensitivity, specificity and clinical potential in breast cancer, such as CK19 (25), mammaglobin (26), HER2 (27), MUC1 (28). The fact that few markers provide adequate sensitivity individually makes combination of markers a good choice for CTCs detec- 
tion $(29,30)$. In the present study, we screened the sensitivity and specificity of CK19, HER2, EpCAM and MUC1 for breast CTCs detection, and found that the real-time RT-PCR assay using KRT19 and ERBB2 as targeted genes could consistently detect a tumor cell in various breast cancer cell lines. In addition, duplexing assay using KRT19 and ERBB2 improved the sensitivity of CTCs detection in breast cancer, especially in early breast cancer compared to that of immunocytochemistry staining.

In summary, our CTC detection approach that combines negative enrichment with real-time quantitative RT-PCR assay using KRT19 and ERBB2 as targeted genes demonstrated high sensitivity, specificity and potential clinical utility in breast cancer.

\section{Acknowledgements}

The present study was supported by the National Natural Science Foundation of China (grant no. 81071434).

\section{References}

1. Ferlay J, Soerjomataram I, Ervik M, et al: GLOBOCAN 2012 v1.0, Cancer Incidence and Mortality Worldwide: IARC CancerBase No. 11. International Agency for Research on Cancer, Lyon, 2013. Last accessed April, 2014.

2. Weigel MT and Dowsett M: Current and emerging biomarkers in breast cancer: prognosis and prediction. Endocr Relat Cancer 17: R245-R262, 2010.

3. Yu M, Stott S, Toner M, Maheswaran S and Haber DA: Circulating tumor cells: approaches to isolation and characterization. J Cell Biol 192: 373-382, 2011.

4. Wallwiener M, Hartkopf AD, Baccelli I, et al: The prognostic impact of circulating tumor cells in subtypes of metastatic breast cancer. Breast Cancer Res Treat 137: 503-510, 2013.

5. Hayes DF, Cristofanilli M, Budd GT, et al: Circulating tumor cells at each follow-up time point during therapy of metastatic breast cancer patients predict progression-free and overall survival. Clin Cancer Res 12: 4218-4224, 2006.

6. Cristofanilli M, Budd GT, Ellis MJ, et al: Circulating tumor cells, disease progression, and survival in metastatic breast cancer. $\mathrm{N}$ Engl J Med 351: 781-791, 2004.

7. Liu Y, Liu Q, Wang T, et al: Circulating tumor cells in HER2positive metastatic breast cancer patients: a valuable prognostic and predictive biomarker. BMC Cancer 13: 202, 2013.

8. Jiang ZF, Cristofanilli M, Shao ZM, et al: Circulating tumor cells predict progression-free and overall survival in Chinese patients with metastatic breast cancer, HER2-positive or triple-negative (CBCSG004): a multicenter, double-blind, prospective trial. Ann Oncol 24: 2766-2772, 2013.

9. Yusa A, Toneri M, Masuda T, et al: Development of a new rapid isolation device for circulating tumor cells (CTCs) using 3D palladium filter and its application for genetic analysis. PLoS One 9: e88821, 2014.

10. Wu S, Liu Z, Liu S, Lin L, Yang W and Xu J: Enrichment and enumeration of circulating tumor cells by efficient depletion of leukocyte fractions. Clin Chem Lab Med 52: 243-251, 2014.

11. Zhao M, Schiro PG, Kuo JS, et al: An automated high-throughput counting method for screening circulating tumor cells in peripheral blood. Anal Chem 85: 2465-2471, 2013.

12. Lee HJ, Cho HY, Oh JH, et al: Simultaneous capture and in situ analysis of circulating tumor cells using multiple hybrid nanoparticles. Biosens Bioelectron 47: 508-514, 2013.
13. Nagrath S, Sequist LV, Maheswaran S, et al: Isolation of rare circulating tumour cells in cancer patients by microchip technology. Nature 450: 1235-1239, 2007.

14. Krishnamurthy S, Cristofanilli M, Singh B, et al: Detection of minimal residual disease in blood and bone marrow in early stage breast cancer. Cancer 116: 3330-3337, 2010.

15. Wu C, Hao H, Li L, et al: Preliminary investigation of the clinical significance of detecting circulating tumor cells enriched from lung cancer patients. J Thorac Oncol 4: 30-36, 2009.

16. Gorges TM, Tinhofer I, Drosch M, et al: Circulating tumour cells escape from EpCAM-based detection due to epithelial-tomesenchymal transition. BMC Cancer 12: 178, 2012.

17. Armstrong AJ, Marengo MS, Oltean S, et al: Circulating tumor cells from patients with advanced prostate and breast cancer display both epithelial and mesenchymal markers. Mol Cancer Res 9: 997-1007, 2011.

18. Yu M, Bardia A, Wittner BS, et al: Circulating breast tumor cells exhibit dynamic changes in epithelial and mesenchymal composition. Science 339: 580-584, 2013.

19. Liu Z, Fusi A, Klopocki E, et al: Negative enrichment by immunomagnetic nanobeads for unbiased characterization of circulating tumor cells from peripheral blood of cancer patients. J Transl Med 9: 70, 2011.

20. Hyun KA, Lee TY and Jung HI: Negative enrichment of circulating tumor cells using a geometrically activated surface interaction chip. Anal Chem 85: 4439-4445, 2013.

21. Sajay BN, Chang CP, Ahmad H, et al: Microfluidic platform for negative enrichment of circulating tumor cells. Biomed Microdevices 6: 537-548, 2014.

22. Yang L, Lang JC, Balasubramanian P, et al: Optimization of an enrichment process for circulating tumor cells from the blood of head and neck cancer patients through depletion of normal cells. Biotechnol Bioeng 102: 521-534, 2009.

23. Lara O, Tong X, Zborowski M and Chalmers JJ: Enrichment of rare cancer cells through depletion of normal cells using density and flow-through, immunomagnetic cell separation. Exp Hematol 32: 891-904, 2004

24. Tong X, Yang L, Lang JC, Zborowski M and Chalmers JJ: Application of immunomagnetic cell enrichment in combination with RT-PCR for the detection of rare circulating head and neck tumor cells in human peripheral blood. Cytometry B Clin Cytom 72: 310-323, 2007.

25. Stathopoulou A, Gizi A, Perraki M, et al: Real-time quantification of CK-19 mRNA-positive cells in peripheral blood of breast cancer patients using the lightcycler system. Clin Cancer Res 9: 5145-5151, 2003.

26. Ntoulia M, Stathopoulou A, Ignatiadis M, et al: Detection of Mammaglobin A-mRNA-positive circulating tumor cells in peripheral blood of patients with operable breast cancer with nested RT-PCR. Clin Biochem 39: 879-887, 2006.

27. Ignatiadis M, Kallergi G, Ntoulia M, et al: Prognostic value of the molecular detection of circulating tumor cells using a multimarker reverse transcription-PCR assay for cytokeratin 19 , mammaglobin A, and HER2 in early breast cancer. Clin Cancer Res 14: 2593-2600, 2008.

28. de Cremoux P, Extra JM, Denis MG, et al: Detection of MUC1expressing mammary carcinoma cells in the peripheral blood of breast cancer patients by real-time polymerase chain reaction. Clin Cancer Res 6: 3117-3122, 2000.

29. Varangot M, Barrios E, Sonora C, et al: Clinical evaluation of a panel of mRNA markers in the detection of disseminated tumor cells in patients with operable breast cancer. Oncol Rep 14: 537-545, 2005.

30. Reinholz MM, Nibbe A, Jonart LM, et al: Evaluation of a panel of tumor markers for molecular detection of circulating cancer cells in women with suspected breast cancer. Clin Cancer Res 11: 3722-3732, 2005. 